Neurosurg Focus 12 (5):Introduction, 2002, Click here to return to Table of Contents

\title{
Introduction to malignant tumors of the skull base
}

\author{
Franco DeMonte, M.D.
}

Department of Neurosurgery, University of Texas, M. D. Anderson Cancer Center, Houston, Texas

Skull base malignancies fortunately affect quite a small number of people. Its rarity is but one of the challenges that these pathologically diverse lesions present to those charged with the management of patients thus afflicted. These tumors often cause symptoms that may be easily attributed to far more frequently occurring illnesses such as sinusitis, otitis media, allergic reactions or Bell palsy, thus making early detection problematic. Even once detected, the challenges continue. The evaluation of the biopsy specimen by an experienced pathologist cannot be overemphasized. Subtle differences need to be appreciated, because optimum management and outcome differ significantly among the varied pathological entities encountered. Fortunately, the advent of skull base surgical technique has improved the surgery-related care of patients with these lesions. The appropriate selection and application of these techniques requires knowledge of the full range of available operative interventions, the biological nature of the tumor, the likelihood of achieving a "margins-negative" resection, and the expected or potential morbidity associated with each approach. Many of these resections would be impossible without the carefully planned and successful use of various reconstructive strategies. Understanding the nuances of tumor location, type, surgical approach, and reconstruction yield substantive contributions to complication avoidance and patient outcome. In this issue of Neurosurgical Focus we hope to highlight some of these nuances. Articles contained herein address issues of early detection, pathological diagnosis, selection of surgical and reconstructive approaches in children and adults, perioperative and anesthetic management, and complication avoidance. I urge those undertaking the treatment of patients with skull base malignancies to contemplate these issues because they can make all the difference to each patient's outcome. 